\title{
Exclusive electroproduction of $\rho_{T}$ meson with twist three accuracy
}

\author{
I. V. Anikin \\ BLTP, JINR, 141980 Dubna, Russia \\ E-mail: anikin@theor.jinr.ru \\ D. Yu. Ivanov \\ Sobolev Institute of Mathematics, 630090 Novosibirsk, Russia \\ E-mail: d-ivanov@math.nsc.ru \\ B. Pire \\ CPhT, Ecole Polytechnique, CNRS, 91128 Palaiseau, France \\ E-mail: pire@cpht.polytechnique.fr
}

\section{Szymanowski*}

SINS, Warsaw, Poland

E-mail: lechszymefuw.edu.pl

\section{S. Wallon}

LPT, Université Paris-Sud, CNRS, 91405 Orsay, France \& UPMC Univ. Paris 06, faculté de physique, 4 place Jussieu, 75252 Paris Cedex 05, France

E-mail: walloneth.u-psud.fr

\begin{abstract}
Exclusive electroproduction of vector mesons is a crucial way to probe QCD factorization properties. Such a factorization is up to now only proven, at the twist 2 level, for a longitudinaly polarized meson. It is crucial to extend our understanding to the case of transversely polarized vector mesons. As a first step in this direction, we evaluate the impact factor of the transition $\gamma^{*} \rightarrow \rho_{T}$, which is the relevant part of the amplitude within the $k_{t}$-factorization approach valid at large energies, taking into account the twist 3 contributions, coming both from quark antiquark and from quark antiquark gluon correlators. We show that a gauge invariant expression is obtained with the help of QCD equations of motion.
\end{abstract}

35th International Conference of High Energy Physics - ICHEP2010,

July 22-28, 2010

Paris France

* Speaker. 


\section{Introduction}

The factorization properties of the leading twist amplitudes allow to study deep exclusive meson electroproduction [1], when the meson is (pseudo)scalar or longitudinally polarized. The case of a transversely polarized vector meson is more involved since the chiral-oddity of its leading twist distribution amplitude (DA) leads to its decoupling in leading twist electroproduction amplitudes [2] unless in reactions with more than two final hadrons [3]. To understand available data [4], one thus needs to consider carefully all twist 3 contributions. The meson quark gluon structure within collinear factorization may be described by Distribution Amplitudes (DAs), classified in [5]. We consider here the case of very high energy collisions at electron proton or electron positron colliders [6,7]. In the literature there are two approaches to the factorization of the scattering amplitudes in exclusive processes at leading and higher twists. The Light-Cone Collinear Factorization (LCCF) [8,9] extends the inclusive approach [10] to exclusive processes, dealing with the factorization in the momentum space around the dominant light-cone direction, while the Covariant Collinear Factorization (CCF) approach in coordinate space was succesfully applied in [5] for a systematic description of DAs of hadrons carrying different twists. We show [11] that these two descriptions are equivalent at twist 3. For that, we perform our analysis within LCCF method in momentum space. It introduces relevant soft correlators which are generally not independent ones. The correlators are reduced to a minimal independent set with the use of equation of motions and of the light-cone-fixing vector independence condition. A dictionary is obtained between LCCF and CCF correlators, proving the equivalence between LCCF and CCF approaches. We illustrate this equivalence by calculating up to twist 3 accuracy within both methods the impact factor $\gamma^{*} \rightarrow \rho_{T}$, which enters the description of the $\gamma^{*} p \rightarrow \rho p$ and $\gamma^{*} \gamma \rightarrow \rho \rho$ processes at large $s$.

\section{LCCF factorization of exclusive processes}

The amplitude for the exclusive process $A \rightarrow \rho B$ is, in the momentum representation and in axial gauge reads ( $H$ and $H_{\mu}$ are 2- and 3-parton coefficient functions, respectively)

$$
\mathscr{A}=\int d^{4} \ell \operatorname{tr}[H(\ell) \Phi(\ell)]+\int d^{4} \ell_{1} d^{4} \ell_{2} \operatorname{tr}\left[H_{\mu}\left(\ell_{1}, \ell_{2}\right) \Phi^{\mu}\left(\ell_{1}, \ell_{2}\right)\right]+\ldots
$$

In (2.1), the soft parts $\Phi$ are the Fourier-transformed 2- or 3-parton correlators which are matrix elements of non-local operators. To factorize the amplitude, we choose the dominant direction around which we decompose our relevant momenta and we Taylor expand the hard part. Let $p \sim p_{\rho}$ and $n$ be two light-cone vectors $(p \cdot n=1)$. Any vector $\ell$ is then expanded as

$$
\ell_{i \mu}=y_{i} p_{\mu}+\left(\ell_{i} \cdot p\right) n_{\mu}+\ell_{i \mu}^{\perp}, \quad y_{i}=\ell_{i} \cdot n,
$$

and the integration measure in (2.1) is replaced as $d^{4} \ell_{i} \longrightarrow d^{4} \ell_{i} d y_{i} \delta\left(y_{i}-\ell \cdot n\right)$. The hard part $H(\ell)$ is then expanded around the dominant $p$ direction:

$$
H(\ell)=H(y p)+\left.\frac{\partial H(\ell)}{\partial \ell_{\alpha}}\right|_{\ell=y p}(\ell-y p)_{\alpha}+\ldots
$$

where $(\ell-y p)_{\alpha} \approx \ell_{\alpha}^{\perp}$ up to twist 3. To obtain a factorized amplitude, one performs an integration by parts to replace $\ell_{\alpha}^{\perp}$ by $\partial_{\alpha}^{\perp}$ acting on the soft correlator. This leads to new operators containing 
transverse derivatives, such as $\bar{\psi} \partial^{\perp} \psi$, thus requiring additional DAs $\Phi^{\perp}(l)$. Factorization is then achieved by Fierz decomposition on a set of relevant Dirac $\Gamma$ matrices, and we end up with

$$
\mathscr{A}=\operatorname{tr}\left[H_{q \bar{q}}(y) \Gamma\right] \otimes \Phi_{q \bar{q}}^{\Gamma}(y)+\operatorname{tr}\left[H_{q \bar{q}}^{\perp \mu}(y) \Gamma\right] \otimes \Phi_{q \bar{q} \mu}^{\perp \Gamma}(y)+\operatorname{tr}\left[H_{q \bar{q} g}^{\mu}\left(y_{1}, y_{2}\right) \Gamma\right] \otimes \Phi_{q \bar{q} g \mu}^{\Gamma}\left(y_{1}, y_{2}\right),
$$

where $\otimes$ is the $y$-integration. Although the fields coordinates $z_{i}$ are on the light-cone in both LCCF and CCF parametrizations of the soft non-local correlators, $z_{i}$ is along $n$ in LCCF while arbitrary in CCF. The transverse physical polarization of the $\rho$-meson is defined by the conditions

$$
e_{T} \cdot n=e_{T} \cdot p=0
$$

Keeping all the terms up to the twist- 3 order with the axial (light-like) gauge, $n \cdot A=0$, the matrix elements of quark-antiquark nonlocal operators for vector and axial-vector correlators without and with transverse derivatives, with $\overleftrightarrow{\partial_{\rho}}=\frac{1}{2}\left(\overrightarrow{\partial_{\rho}}-\overleftarrow{\partial_{\rho}}\right)$, can be written as (here, $z=\lambda n$ )

$$
\begin{aligned}
\left\langle\rho\left(p_{\rho}\right)\left|\bar{\psi}(z) \gamma_{\mu} \psi(0)\right| 0\right\rangle & =m_{\rho} f_{\rho} \int_{0}^{1} d y \exp [i y p \cdot z]\left[\varphi_{1}(y)\left(e^{*} \cdot n\right) p_{\mu}+\varphi_{3}(y) e_{T \mu}^{*}\right] \\
\left\langle\rho\left(p_{\rho}\right)\left|\bar{\psi}(z) \gamma_{\mu} i \overleftrightarrow{\partial_{\alpha}^{T}} \psi(0)\right| 0\right\rangle & =m_{\rho} f_{\rho} \int_{0}^{1} d y \exp [i y p \cdot z] \varphi_{1}^{T}(y) p_{\mu} e_{T \alpha}^{*} \\
\left\langle\rho\left(p_{\rho}\right)\left|\bar{\psi}(z) \gamma_{5} \gamma_{\mu} \psi(0)\right| 0\right\rangle & =m_{\rho} f_{\rho} i \int_{0}^{1} d y \exp [i y p \cdot z] \varphi_{A}(y) \varepsilon_{\mu \alpha \beta \delta} e_{T}^{* \alpha} p^{\beta} n^{\delta} \\
\left\langle\rho\left(p_{\rho}\right)\left|\bar{\psi}(z) \gamma_{5} \gamma_{\mu} i \overleftrightarrow{\partial_{\alpha}^{T}} \psi(0)\right| 0\right\rangle & =m_{\rho} f_{\rho} i \int_{0}^{1} d y \exp [i y p \cdot z] \varphi_{A}^{T}(y) p_{\mu} \varepsilon_{\alpha \lambda \beta \delta} e_{T}^{* \lambda} p^{\beta} n^{\delta}
\end{aligned}
$$

where $y(\bar{y})$ is the quark (antiquark) momentum fraction. Two analogous correlators are needed to describe gluonic degrees of freedom, introducing $B$ and $D$ DAs according to

$$
\begin{gathered}
\left\langle\rho\left(p_{\rho}\right)\left|\bar{\psi}\left(z_{1}\right) \gamma_{\mu} g A_{\alpha}^{T}\left(z_{2}\right) \psi(0)\right| 0\right\rangle=m_{\rho} f_{3 \rho}^{V} \int_{0}^{1} d y_{1} \int_{0}^{1} d y_{2} e^{i y_{1} p \cdot z_{1}+i\left(y_{2}-y_{1}\right) p \cdot z_{2}} B\left(y_{1}, y_{2}\right) p_{\mu} e_{T \alpha}^{*}, \\
\left\langle\rho\left(p_{\rho}\right)\left|\bar{\psi}\left(z_{1}\right) \gamma_{5} \gamma_{\mu} g A_{\alpha}^{T}\left(z_{2}\right) \psi(0)\right| 0\right\rangle=m_{\rho} f_{3 \rho}^{A} \int_{0}^{1} d y_{1} \int_{0}^{1} d y_{2} e^{i y_{1} p \cdot z_{1}+i\left(y_{2}-y_{1}\right) p \cdot z_{2}} i D\left(y_{1}, y_{2}\right) \\
\times p_{\mu} \varepsilon_{\alpha \lambda \beta \delta} e_{T}^{* \lambda} p^{\beta} n^{\delta} .
\end{gathered}
$$

One thus needs 7 DAs: $\varphi_{1}$ (twist-2), $B$ and $D$ (genuine (dynamical) twist-3) and $\varphi_{3}, \varphi_{A}, \varphi_{1}^{T}, \varphi_{A}^{T}$ (kinematical (à la Wandzura-Wilczek) twist-3 and genuine (dynamical) twist-3).

These DAs are not independent. They are related by 2 Equations of Motions (EOMs) and 2 equations arising from the invariance of $\mathscr{A}$ under the arbitrary vector $n$, which comes from 3 sources. First, it enters the definition of the non-local correlators through the light-like separation $z=\lambda n$. These correlators are defined in the axial light-like gauge $n \cdot A=0$, which allows to get rid of Wilson lines. Second, it determines the notion of transverse polarization of the $\rho$. Last, $n$ enters the Sudakov decomposition (2.2) which defines the transverse parton momentum involved in the collinear factorization. One can in fact show that the hard part does not depend on the gauge fixing vector $n$. Therefore, only the second and third source of $n$-dependence should be investigated. Based on Ward identities, this $n$-dependence of $\mathscr{A}$ can be recast in a system of constraints which only involve the soft part. We thus have only 3 independent DAs $\varphi_{1}, B$ and $D$, which fully encode 
the non-perturbative content of the $\rho$ at twist 3 .

The original CCF parametrizations of the $\rho$ DAs [5] also involve 3 independent DAs, defined through 4 correlators related by EOMs. The 2-parton axial-vector correlator reads,

$$
\left\langle\rho\left(p_{\rho}\right)\left|\bar{\psi}(z)[z, 0] \gamma_{\mu} \gamma_{5} \psi(0)\right| 0\right\rangle=\frac{1}{4} f_{\rho} m_{\rho} \varepsilon_{\mu}^{\alpha \beta \gamma} e_{T \alpha}^{*} p_{\beta} z_{\gamma} \int_{0}^{1} d y e^{i y(p \cdot z)} g_{\perp}^{(a)}(y),
$$

$\left[z_{1}, z_{2}\right]=P \exp \left[i g \int_{0}^{1} d t\left(z_{1}-z_{2}\right) \mu A^{\mu}\left(t z_{1}+(1-t) z_{2}\right)\right]$ being the Wilson line. Denoting the meson polarization vector by $e, e_{T}$ is here defined to be orthogonal to the light-cone vectors $p$ and $z$ :

$$
e_{T \mu}=e_{\mu}-p_{\mu} \frac{e \cdot z}{p \cdot z}-z_{\mu} \frac{e \cdot p}{p \cdot z} .
$$

Thus $e_{T}$ (2.13) in CCF and $e_{T}$ (2.5) in LCCF differ since $z$ does not generally point in the $n$ direction. The 2-parton vector correlator reads (up to twist 3)

$$
\left\langle\rho\left(p_{\rho}\right)\left|\bar{\psi}(z)[z, 0] \gamma_{\mu} \psi(0)\right| 0\right\rangle=f_{\rho} m_{\rho} \int_{0}^{1} d y e^{i y(p \cdot z)}\left[p_{\mu} \frac{e^{*} \cdot z}{p \cdot z} \phi_{\|}(y)+e_{T \mu}^{*} g_{\perp}^{(v)}(y)\right] .
$$

The 3-parton correlators are parametrized (up to twist 3 level) according to

$$
\begin{aligned}
\left\langle\rho\left(p_{\rho}\right)\left|\bar{\psi}(z)[z, t z] \gamma_{\alpha} g G_{\mu v}(t z)[t z, 0] \psi(0)\right| 0\right\rangle & =-i p_{\alpha}\left[p_{\mu} e_{\perp v}^{*}-p_{v} e_{\perp \mu}^{*}\right] m_{\rho} f_{3 \rho}^{V} \\
& \times \int D \alpha V\left(\alpha_{1}, \alpha_{2}\right) e^{i p \cdot z\left(\alpha_{1}+t \alpha_{g}\right)} \\
\left\langle\rho\left(p_{\rho}\right)\left|\bar{\psi}(z)[z, t z] \gamma_{\alpha} \gamma_{5} g \tilde{G}_{\mu v}(t z)[t z, 0] \psi(0)\right| 0\right\rangle & =-p_{\alpha}\left[p_{\mu} e_{\perp v}^{*}-p_{v} e_{\perp \mu}^{*}\right] m_{\rho} f_{3 \rho}^{A} \\
& \times \int D \alpha A\left(\alpha_{1}, \alpha_{2}\right) e^{i p \cdot z\left(\alpha_{1}+t \alpha_{g}\right)}
\end{aligned}
$$

where $\alpha_{1}, \alpha_{2}, \alpha_{g}$ are momentum fractions of quark, antiquark and gluon respectively inside the $\rho-$ meson, $\int D \alpha=\int_{0}^{1} d \alpha_{1} \int_{0}^{1} d \alpha_{2} \int_{0}^{1} d \alpha_{g} \delta\left(1-\alpha_{1}-\alpha_{2}-\alpha_{g}\right)$ and $\tilde{G}_{\mu \nu}=-\frac{1}{2} \varepsilon_{\mu \nu \alpha \beta} G^{\alpha \beta}$. A comparison of the correlators $(2.6,2.7,2.8,2.9,2.10,2.11)$ and $(2.12,2.14,2.15,2.16)$ in the axial gauge $n \cdot A=$ 0 gives the following identification of the 2- and 3-parton DAs in LCCF and CCF approaches:

$$
\begin{aligned}
& \varphi_{1}(y)=\phi_{\|}(y), \quad \varphi_{3}(y)=g_{\perp}^{(v)}(y), \quad \varphi_{A}(y)=-\frac{1}{4} \frac{\partial g_{\perp}^{(a)}(y)}{\partial y}, \\
& B\left(y_{1}, y_{2}\right)=-\frac{V\left(y_{1}, 1-y_{2}\right)}{y_{2}-y_{1}} \quad D\left(y_{1}, y_{2}\right)=-\frac{A\left(y_{1}, 1-y_{2}\right)}{y_{2}-y_{1}} .
\end{aligned}
$$

\section{3. $\gamma^{*} \rightarrow \rho_{T}$ Impact factor up to twist three accuracy in LCCF and CCF}

We have calculated, in both LCCF and CCF, the forward impact factor $\Phi^{\gamma^{*} \rightarrow \rho}$ of the subprocess $g+\gamma^{*} \rightarrow g+\rho_{T}$, defined as the integral of the discontinuity in the $s$ channel of the off-shell S-matrix element $\mathscr{S}_{\mu}^{\gamma_{T}^{*} g \rightarrow \rho_{T} g}$. In LCCF, one computes the diagrams perturbatively in a fairly direct way, which makes the use of the CCF parametrization [5] less practical. We need to express the impact factor in terms of hard coefficient functions and soft parts parametrized by the lightcone matrix elements. The standard technique here is an operator product expansion on the light cone, which gives the leading term in the power counting. Since there is no operator definition for an impact factor, we have to rely on perturbation theory. The primary complication encountered is 
that the $z^{2} \rightarrow 0$ limit of any single diagram is given in terms of light-cone matrix elements without any Wilson line insertion between the quark and gluon operators ("perturbative correlators"), like $\left\langle\rho\left(p_{\rho}\right)\left|\bar{\psi}(z) \gamma_{\mu} \psi(0)\right| 0\right\rangle$. Despite working in the axial gauge one cannot neglect effects coming from the Wilson lines since the two light cone vectors $z$ and $n$ are not identical and thus, generically, Wilson lines are not equal to unity. Nevertheless in the axial gauge the contribution of each additional parton costs one extra power of $1 / Q$, allowing the calculation to be organized in a simple iterative manner expanding the Wilson line. At twist 3, we need to keep the contribution $[z, 0]=1+i g \int_{0}^{1} d t z^{\alpha} A_{\alpha}(z t)$ and to care about the difference between the physical $\rho_{T}$-polarization (2.5) from the formal one (2.13). At twist 3-level the net effect of the Wilson line when computing our impact factor is just a renormalization of the DA $g_{\perp}^{a}$ of (2.12), and similarly for the vector case. We are then able to show that our two LCCF and CCF results are identical; the result is gauge invariant due to a consistent inclusion of fermionic and gluonic degrees of freedom and it is free of end-point singularities, due to the $k_{T}$ regulator.

This establishes a consistent gauge invariant analysis of electroproduction of transversely polarized vector mesons at high energy. An extension of this work to lower energy regime where collinear factorization allows to write the amplitude in terms of generalized parton distributions is under way.

This work is partly supported by the grant ANR-06-JCJC-0084, the RFBR (grants 09-0201149, 09-02-00263, 08-02-00896), the grant NSh-3810.2010.2 and the Polish Grant N202 249235.

\section{References}

[1] J. C. Collins, L. Frankfurt, M. Strikman, Phys. Rev. D 56, 2982 (1997).

[2] M. Diehl et al., Phys. Rev. D 59, 034023 (1999); J. C. Collins et al., Phys. Rev. D 61, 114015 (2000).

[3] D. Yu. Ivanov et al., Phys. Lett. B 550, 65 (2002); R. Enberg et al., Eur. Phys. J. C 47, 87 (2006); M. E. Beiyad et al., arXiv:0911.2611; Phys. Lett. B 688, 154 (2010); arXiv:1006.0740.

[4] S. A. Morrow et al. [CLAS], Eur. Phys. J. A 39, 5 (2009); A. Borissov [HERMES], AIP Conf. Proc. 1105 (2009) 19; V. Y. Alexakhin et al. [COMPASS], Eur. Phys. J. C 52, 255 (2007); F. D. Aaron et al. [H1], JHEP 1005, 032 (2010); J. Breitweg et al. [ZEUS], Eur. Phys. J. C 6, 603 (1999).

[5] P. Ball et al., Phys. Rev. D 54, 2182 (1996); Nucl. Phys. B 529, 323 (1998); ibid. B 543, 201 (1999).

[6] D. Yu. Ivanov et al., Eur. Phys. J. C 38, 195 (2004) and 49, 947 (2007); Nucl. Phys. B 732, 183 (2006).

[7] B. Pire et al., Eur. Phys. J. C 44, 545 (2005); Phys. Lett. B 639, 642 (2006); R. Enberg et al. Eur. Phys. J. C 45, 759 (2006) [Erratum-ibid. C 51, 1015 (2007)]; M. Segond et al. Eur. Phys. J. C 52, 93 (2007).

[8] I. V. Anikin and O. V. Teryaev, Phys. Lett. B 554, 51 (2003); Phys. Lett. B 509, 95 (2001).

[9] I. V. Anikin, B. Pire and O. V. Teryaev, Phys. Rev. D 62, 071501 (2000).

[10] A. V. Efremov et al., Sov. J. Nucl. Phys. 36, 140 (1982) [Yad. Fiz. 36, 242 (1982)]; E. V. Shuryak et al., Nucl. Phys. B 199, 451 (1982), Nucl. Phys. B 201, 141 (1982); R.K. Ellis et al., Nucl. Phys. B 212, 29 (1983); A.V. Efremov et al., Sov. J. Nucl. Phys. 39, 962 (1984); O. V. Teryaev, arXiv:hep-ph/0102296; A. V. Radyushkin et al., Phys. Rev. D 64, 097504 (2001).

[11] I. V. Anikin et al., AIP Conf. Proc. 1105, 390 (2009); Phys. Lett. B 682, 413 (2010); Acta Phys. Polon. B 40, 2131 (2009); arXiv:0909.4038; arXiv:0909.4042; arXiv:1006.1477; Nucl. Phys. B 828, 1 (2010). 